\title{
Modelado y control de una grúa móvil con tres grados de libertad
}

\section{Modeling and control of a mobile crane with three degrees of freedom}

\author{
Gerardo Bonilla Mota ${ }^{1}$ (i) y Elizabeth Guevara \\ Facultad de Ingeniería, Universidad Anáhuac México, México
}

Resumen. En la industria, el traslado de material pesado en espacios pequeños requiere de grúas en las que el control debe ser preciso, ya que de lo contrario se pueden producir balanceos de la carga que pueden dañar tanto a los materiales transportados como al equipo que lo rodea, de ahí la importancia del control. El objetivo de este trabajo es el modelado de un sistema mecánico tipo grúa de tres grados de libertad constituido por un carro sobre una plataforma horizontal y un péndulo doble. Así como la propuesta de una ley de control que garantice que las oscilaciones que se presentan en la carga cuando la grúa se desplaza de un punto a otro se cancelen o minimicen. El modelo dinámico del sistema se obtuvo mediante ecuaciones diferenciales ordinarias no lineales y la ley de control propuesta se basa en un regulador cuadrático lineal (LQR - Linear Quadratic Regulator) con un prefiltro el cual permite reducir las oscilaciones en el traslado de la carga con respecto a un control LQR sin prefiltro.

Palabras Claves. Ecuaciones diferenciales no lineales; control LQR; prefiltro; péndulo doble.

Resumen. In the industrial sector, the transportation of heavy material in small spaces requires cranes with a very precise control, otherwise load swing can occur and that can damage both, transported materials and the surrounding environment, therefore control is very important. The purpose of this work is the modeling of a mechanical crane system of three degrees of freedom constituted by a trolley on a horizontal platform and a double pendulum. As well as the proposal of a control law that guarantees that the oscillations, that occur in the load when the crane moves from one point to another, are canceled or minimized. The dynamic model of the system was obtained through non-linear ordinary differential equations and the proposed control law is based on a linear quadratic regulator (LQR) with a prefilter that allows to reduce the oscillations during the load displacement with respect to an LQR control without prefilter.

Keywords. Nonlinear differential equations; LQR control; prefilter; double pendulum.

Como citar. G. Bonilla Mota y E. Guevara, "Modelado y control de una grúa móvil con tres grados de libertad", Jou. Cie. Ing., vol. 12, no. 1, pp. 127-137, 2020. doi:10.46571/JCI.2020.1.12

Recibido: 16/10/2019 Revisado: 06/04/2020 Aceptado: 15/05/2020

\section{Introducción}

Los sistemas de control automático son de gran importancia en diferentes áreas de la ingeniería o la física aplicada. La teoría de control moderno y la práctica del control automático han

1 gerardo.bonillam@anahuac.mx 
proporcionado los medios para mejorar la productividad, logrando el funcionamiento óptimo de sistemas dinámicos, obteniendo como resultado tiempos menores de producción, mayor seguridad en los sistemas de alto riesgo, etc.

En el caso de las grúas, éstas se han utilizado para levantar y transportar materiales pesados de un punto a otro en diferentes lugares como fábricas, almacenes, sitios de construcción y astilleros, entre otros. Sin embargo, debido a su estructura, la carga tiende a oscilar durante las operaciones de transporte por lo que el control de estas oscilaciones es importante para asegurar tanto la precisión al dejar la carga como la seguridad al evitar colisiones con objetos o personas $[1,2]$.

En general, las grúas se pueden dividir en grúas aéreas (puente o viajeras), fijas o móviles. En las categorías de fijas y móviles también hay subdivisiones, por ejemplo, las grúas fijas pueden ser grúas torre, telescópicas, pórtico, etc. Entre las grúas móviles se encuentran las grúas montadas en camiones, grúas de plataforma, flotantes, entre muchas otras. Este trabajo se enfoca en una grúa viajera.

El modelado de los sistemas de control es una de las partes más importantes en la ingeniería de control. Este modelo ayuda a entender teórica y físicamente el comportamiento de los sistemas, así como a elegir y diseñar el algoritmo de control apropiado con base en el problema que se quiere resolver. Las grúas presentan propiedades interesantes para el control desde el punto de vista de la ingeniería. Éstas son consideradas como sistemas mecánicos subactuados, esto es, el número de actuadores es menor que el número de grados de libertad [3] lo que hace que sean difíciles de controlar debido a su dinámica compleja principalmente cuando también tienen un comportamiento no holonómico.

En [4] se realiza una revisión y un estudio detallado de los desafíos en el modelado y el control de los sistemas de grúas. Los autores establecen que para simplificar las complejidades de tratar con un modelo no lineal como el de la grúa, en muchos trabajos se hacen aproximaciones linealizadas. Esta simplificación implica una reducción de la robustez del controlador. Sin embargo, mencionan que los modelos simplificados - como el utilizado en este trabajo- siguen siendo una buena aproximación y son útiles en condiciones de carga especiales.

Con respecto al control, se tienen esquemas de control de lazo abierto $[5,6]$ y de lazo cerrado [7-14]. En [15] se presenta una investigación de las estrategias de control aplicadas a sistemas de grúas, estableciendo la importancia del problema.

El objetivo general de este trabajo es diseñar una ley de control para una grúa viajera de doble péndulo, que garantice que las oscilaciones que se presenten en la carga cuando la grúa se traslade de un punto inicial a otro final se cancelen o minimicen. El comportamiento de la grúa se representa mediante un modelo matemático descrito por ecuaciones diferenciales ordinarias no lineales, que se linealiza alrededor de un punto de equilibrio. Con base en este modelo linealizado se propone una estrategia de control usando LQR y se calculan los parámetros de un prefiltro o precompensador para aplicarlo al sistema y realizar comparaciones. Es importante mencionar que el desempeño del sistema retroalimentado se evalúa sobre el modelo no-lineal original y que el problema de controlar un péndulo doble no es trivial ya que su movimiento es caótico.

\section{Modelo matemático de una grúa con tres grados de libertad}

La grúa viajera está constituida por un carro en movimiento sobre una plataforma horizontal y un péndulo doble. El objetivo es determinar un modelo lineal invariante en el tiempo para este mecanismo. El modelo no lineal se obtiene aplicando el método de Euler-Lagrange [16]. Posteriormente, la linealización se hace alrededor de un punto de equilibrio.

Para obtener el modelo dinámico no lineal de la grúa viajera se realizan las siguientes consideraciones: el sistema se mueve en un plano, el cable se considera rígido y recto con longitud constante y masa despreciable y la carga, que se encuentra en el extremo terminal del cable, se considera como una masa puntual $m$. También se establece que no existe un coeficiente 
G. Bonilla Mota, et al. $\quad$ Modelado y control de una grúa móvil con tres grados de libertad...

de amortiguamiento para la grúa.

Considérese el sistema subactuado con tres grados de libertad que se muestra en la figura 1 , donde $M$ es la masa del carro, $m_{1}$ es la masa del primer segmento, $\theta_{1}$ es el ángulo que el primer segmento forma con la vertical y $l_{1}$ es la distancia que existe entre el punto de enlace del carro y el primer péndulo, $m_{2}$ es la masa del segundo segmento, $\theta_{2}$ es el ángulo que forma el segundo segmento con la vertical y $l_{2}$ es la longitud del segundo segmento, $f$ es la fuerza externa que se aplica al carro, $f_{r}$ es una fuerza virtual aplicada en el punto de unión de los cables y $x$ es el desplazamiento del carro en el plano horizontal. Se asume que las masas se concentran en los extremos finales de cada segmento.

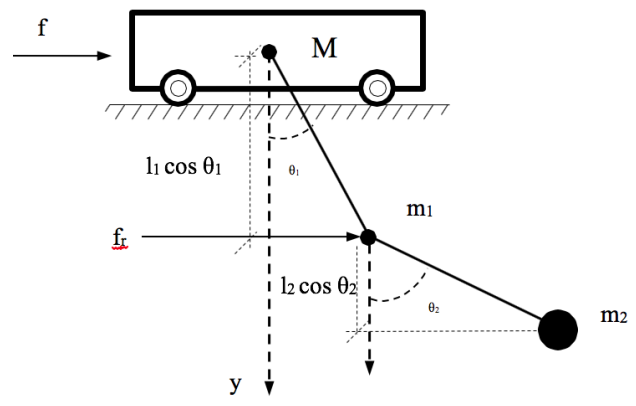

Figura 1: Sistema de grúa con dos segmentos

La masa $M$ del carro tiene como coordenadas $x_{c}=x, y_{c}=0$, la masa $m_{1}$ tiene las siguientes coordenadas:

$$
\begin{gathered}
x_{1 G}=x+l_{1} \sin \theta_{1}, \\
y_{1 G}=-l_{1} \cos \theta_{1}
\end{gathered}
$$

y las coordenadas de la masa $m_{2}$ son:

$$
\begin{aligned}
& x_{2 G}=x+l_{1} \sin \theta_{1}+l_{2} \sin \theta_{2}, \\
& y_{2 G}=-l_{1} \cos \theta_{1}-l_{2} \cos \theta_{2}
\end{aligned}
$$

Por tanto, el Lagrangiano del sistema, donde $K$ es la energía cinética y $P$ es la energía potencial, es:

$$
\begin{aligned}
L= & K-P, \\
= & \frac{1}{2}\left(M+m_{1}+m_{2}\right) \dot{x}^{2}+\dot{x}\left(m_{1}+m_{2}\right) l_{1} \cos \theta_{1} \dot{\theta}_{1} \\
& +\dot{x} m_{2} l_{2} \cos \theta_{2} \dot{\theta}_{2}+\frac{1}{2}\left(m_{1}+m_{2}\right) l_{1}^{2} \dot{\theta}_{1}^{2} \\
& +m_{2} l_{1} l_{2} \cos \left(\theta_{1}-\theta_{2}\right) \dot{\theta}_{1} \dot{\theta}_{2}+\frac{1}{2} m_{2} l_{2}^{2} \dot{\theta}_{2}^{2} \\
& +\left(m_{1}+m_{2}\right) g l_{1} \cos \theta_{1}+m_{2} g l_{2} \cos \theta_{2} .
\end{aligned}
$$

Las ecuaciones de Euler-Lagrange se definen como:

$$
\frac{d}{d t}\left[\frac{\partial L}{\partial \dot{q}_{i}}\right]-\frac{\partial L}{\partial q_{i}}=\tau_{i}, \quad i=1,2, \ldots, n,
$$

nonebbl@id@@spanishid@@spanishdonde $q_{i}=\left(q_{1}, \ldots, q_{n}\right)^{T}$ representan las variables generalizadas, una para cada grado de libertad del sistema, $L$ es el Lagrangiano y $\tau=\left(\tau_{1}, \ldots, \tau_{n}\right)^{T}$ denotan las fuerzas generalizadas que externamente son aplicadas al sistema.

La dinámica del sistema se puede expresar mediante la ecuación estándar siguiente:

$$
\tau=M(q) \ddot{q}+C(q, \dot{q}) \dot{q}+g(q)
$$


G. Bonilla Mota, et al. $\quad$ Modelado y control de una grúa móvil con tres grados de libertad...

nonebbl@id@@spanishid@@spanishdonde $M(q)$ es definida como la matriz de Inercia, la cual tiene la particularidad de ser una matriz simétrica definida positiva, $C(q, \dot{q})$ es la matriz de fuerzas de centrípetas y de Coriolis y $g(q)$ es el vector de fuerzas gravitacionales.

Obteniendo las ecuaciones de Euler-Lagrange (4) para cada uno de los tres grados de libertad y expresando la dinámica del sistema mediante la ecuación (5), se obtienen los siguientes componentes:

$$
\begin{gathered}
q=\left[\begin{array}{c}
x \\
\theta_{1} \\
\theta_{2}
\end{array}\right] y \tau=\left[\begin{array}{l}
f \\
0 \\
0
\end{array}\right], \\
M(q)=\left[\begin{array}{ccc}
M+m_{1}+m_{2} & \left(m_{1}+m_{2}\right) l_{1} \cos \theta_{1} & m_{2} l_{2} \cos \theta_{2} \\
\left(m_{1}+m_{2}\right) l_{1} \cos \theta_{1} & \left(m_{1}+m_{2}\right) l_{1}^{2} & m_{2} l_{1} l_{2} \cos \left(\theta_{2}-\theta_{1}\right) \\
m_{2} l_{2} \cos \theta_{2} & m_{2} l_{1} l_{2} \cos \left(\theta_{2}-\theta_{1}\right) & m_{2} l_{2}^{2}
\end{array}\right], \\
C(q, \dot{q})=\left[\begin{array}{ccc}
0 & -\left(m_{1}+m_{2}\right) l_{1} \sin \theta_{1} \dot{\theta}_{1} & -m_{2} l_{2} \sin \theta_{2} \dot{\theta}_{2} \\
0 & 0 & -m_{2} l_{1} l_{2} \sin \left(\theta_{2}-\theta_{1}\right) \dot{\theta}_{2} \\
0 & m_{2} l_{1} l_{2} \sin \left(\theta_{2}-\theta_{1}\right) \dot{\theta}_{1} & 0
\end{array}\right], \\
g(q)=\left[\begin{array}{cc}
\left(m_{1}+m_{2}\right) g l_{1} \sin \theta_{1} \\
m_{2} g l_{2} \sin \theta_{2}
\end{array}\right]
\end{gathered}
$$

Para facilitar la aplicación de las técnicas de control lineal se realiza un cambio de variables para obtener el modelo en variables de estado y linealizar el sistema no lineal alrededor del punto de equilibrio para el caso trivial $\left(x, \dot{x}, \theta_{1}, \dot{\theta}_{1}, \theta_{2}, \dot{\theta}_{2}\right)=(0,0,0,0,0,0)$, mediante una expansión en serie de Taylor del modelo en variables de estado. Tomando la primera aproximación, se obtiene un sistema de la forma:

$$
\begin{aligned}
& \dot{x}=A x+B u, \\
& y=C x .
\end{aligned}
$$

donde:

$$
\begin{aligned}
& \begin{array}{c}
A=\left.\frac{\partial f(x)}{\partial x}\right|_{x=0}= \\
=\left[\begin{array}{cccccc}
0 & 1 & 0 & 0 & 0 & 0 \\
0 & 0 & \frac{\left(m_{1}+m_{2}\right) g}{M} & 0 & 0 & 0 \\
0 & 0 & 0 & 1 & 0 & 0 \\
0 & 0 & \frac{-\left(m_{1}+m_{2}+M\left(1+m_{2}\right)\right) g}{l_{1} M} & 0 & \frac{m_{2} g}{l_{1} m_{1}} & 0 \\
0 & 0 & 0 & 0 & 0 & 1 \\
0 & 0 & \frac{\left(m_{1}+m_{2}\right) g}{l_{2} m_{1}} & 0 & \frac{-\left(m_{1}+m_{2}\right) g}{l_{2} m_{1}} & 0
\end{array}\right],
\end{array} \\
& B=\left.\frac{\partial g(x)}{\partial x}\right|_{x=0}=\left[\begin{array}{c}
0 \\
\frac{1}{M} \\
0 \\
\frac{-1}{l_{1} M} \\
0 \\
0
\end{array}\right] \\
& C=\left.\frac{\partial h(x)}{\partial x}\right|_{x=0}=\left[\begin{array}{llllll}
1 & 0 & 0 & 0 & 0 & 0 \\
0 & 1 & 0 & 0 & 0 & 0
\end{array}\right] \text {. }
\end{aligned}
$$


G. Bonilla Mota, et al. $\quad$ Modelado y control de una grúa móvil con tres grados de libertad...

El análisis de estabilidad del punto de equilibrio del sistema lineal se deduce directamente de la localización de los valores propios de la matriz $A$. El cálculo de los eigenvalores, utilizando los siguientes parámetros: $M=100, m_{1}=1, m_{2}=100, l_{1}=1, l_{2}=1, g=10$, es:

$$
\begin{aligned}
& p_{1,2}=0, \\
& p_{3,4}=0 \pm 44.9450 i, \\
& p_{5,6}=0 \pm 3.1701 i .
\end{aligned}
$$

La existencia de estos polos indica que el punto de equilibrio, considerado para el sistema no lineal, no es asintóticamente estable sino que tiene un comportamiento polinomialmente inestable.

\section{Diseño de la ley de control}

La ley de control que se analiza es el regulador cuadrático lineal LQR aplicado al modelo linealizado. Con base en el sistema lineal en la forma de variables de estado se construye una ley de retroalimentación de estado que tiene la siguiente forma:

$$
u(t)=-k x,
$$

donde el vector de ganancias $k=\left[\begin{array}{llllll}k_{1} & k_{2} & k_{3} & k_{4} & k_{5} & k_{6}\end{array}\right]$ y $x$ es el vector de estado. Sustituyendo la ley de control (13) en (10) se obtiene:

$$
\dot{x}=(A-B k) x .
$$

Una manera para obtener las ganancias de retroalimentación es tratando de optimizar el proceso mediante la teoría de control óptimo. Considérese que el objetivo es minimizar la siguiente función de costo:

$$
J=\int_{0}^{\infty}\left(x^{T} Q x+u^{T} R u\right) d t,
$$

donde $Q$ es una matriz simétrica semidefinida positiva que pondera los estados y $R$ es un escalar positivo que pondera la entrada. $Q$ y $R$ se seleccionan para tener un control lineal óptimo tal que:

$$
k=R^{-1} B^{T} P,
$$

donde $P$ es la matriz simétrica definida positiva de $n \times n$ que soluciona la ecuación de Riccatti [17]:

$$
A^{T} P+P A-P B R^{-1} B^{T} P+Q=0
$$

Sustituyendo los siguientes valores en (11), $m_{1}=1, m_{2}=100, M=100, l_{1}=1, l_{2}=1, g=10$, las matrices para la grúa viajera quedan como: 


$$
\begin{aligned}
A & =\left[\begin{array}{cccccc}
0 & 1 & 0 & 0 & 0 & 0 \\
0 & 0 & 10.1 & 0 & 0 & 0 \\
0 & 0 & 0 & 1 & 0 & 0 \\
0 & 0 & -1020.1 & 0 & 1000 & 0 \\
0 & 0 & 0 & 0 & 0 & 1 \\
0 & 0 & 10.1 & 0 & -10.1 & 0
\end{array}\right] \\
B & =\left[\begin{array}{c}
0 \\
\frac{1}{100} \\
0 \\
\frac{-1}{100} \\
0 \\
0
\end{array}\right]
\end{aligned}
$$

Como el par $(A, B)$ es controlable, entonces cualquier retroalimentación de estado de la forma $u=-k x$ que estabiliza asintóticamente la aproximación lineal, también estabiliza asintóticamente el sistema no lineal original en una vecindad alrededor del punto de equilibrio en cuestión [18]. Por tanto, las ecuaciones no lineales originales tienen estabilidad local en el punto de equilibrio.

Se proponen valores de elementos de $Q$ que ponderan más a las velocidades angulares $\dot{\theta}_{1}$ y $\dot{\theta}_{2}$ con el fin de tener oscilaciones pequeñas y se propone el siguiente valor de $R$ :

$$
\begin{aligned}
Q & =\left[\begin{array}{cccccc}
1 & 0 & 0 & 0 & 0 & 0 \\
0 & 10 & 0 & 0 & 0 & 0 \\
0 & 0 & 10 & 0 & 0 & 0 \\
0 & 0 & 0 & 100 & 0 & 0 \\
0 & 0 & 0 & 0 & 10 & 0 \\
0 & 0 & 0 & 0 & 0 & 100
\end{array}\right] \\
R & =[1]
\end{aligned}
$$

Considerando lo anterior, el vector de retroalimentación $k$ que se obtiene es:

$$
k=\left[\begin{array}{llllll}
1.0000 & 20.2999 & -1.4474 & -4.1842 & 1.0149 & 9.8568
\end{array}\right],
$$

y la ubicación de los polos en lazo cerrado es:

$$
\begin{aligned}
& p_{1,2}=-0.0505 \pm 0.0493 i \\
& p_{3,4}=-0.0355 \pm 44.9450 i \\
& p_{5,6}=-0.0364 \pm 3.1699 i .
\end{aligned}
$$

Comparando estos valores característicos en malla cerrada contra los correspondientes en malla abierta (12) se puede observar que las partes imaginarias de $p_{3}$ a $p_{6}$ se mantienen prácticamente iguales a los de malla abierta. Esto implica que la naturaleza oscilatoria del sistema original se preserva en el sistema retroalimentado, a pesar de haber sido estabilizado, con lo que se asegura que el sistema tiene un comportamiento asintóticamente estable.

\section{Prefiltros}

El precompensador o prefiltro tiene como objetivo reducir los transitorios en el sistema global de control [19]. Al estar en cascada con el sistema, no tiene ningún efecto sobre la estabilidad 
G. Bonilla Mota, et al. $\quad$ Modelado y control de una grúa móvil con tres grados de libertad...

o la atenuación de perturbaciones aditivas a la salida; y su único objetivo es el de disminuir la respuesta transitoria. Después del diseño de un controlador retroalimentado que satisface requerimientos de estabilidad y/o atenuación de perturbaciones, se agrega un prefiltro que acondicione adecuadamente la señal de referencia para reducir la respuesta transitoria.

Sea la función de transferencia de segundo orden:

$$
h(s)=\frac{w^{2}}{s^{2}+2 \delta w s+w^{2}} ; \quad \delta \in[0,1) .
$$

Aplicando una entrada $u(s)=\frac{A}{s}+\frac{(1-A) e^{-s T}}{s}$, la respuesta es:

$$
\begin{gathered}
y(t)=\left[A-A e^{-\delta w t} \cos \left(w_{r} t\right)-\frac{2 \delta e^{-\delta w t}}{\sqrt{1-\delta^{2}}} \sin \left(w_{r} t\right)\right] \cdot 1(t) \\
+1(t-T)\{(1-A) \\
-(1-A) e^{-\delta w(t-T)} \cos \left(w_{r}(t-T)\right) \\
\left.-\frac{2 \delta(1-A) e^{-\delta w(t-T)}}{\sqrt{1-\delta^{2}}} \sin \left(w_{r}(t-T)\right)\right\}
\end{gathered}
$$

donde $1(t)$ representa un escalón unitario aplicado en $t=0$. Definiendo $T$ como el tiempo en que ocurre el sobrepaso:

$$
T=\frac{\pi}{\sqrt{1-\delta^{2}} w}
$$

e igualando los coeficientes de $\cos \left(w_{r} t\right)$ y $\cos \left(w_{r}(t-T)\right)$ se obtiene:

$$
(1-A) \exp (-\delta w(t-T))=A \exp (-\delta w t)
$$

donde,

$$
A\left(1+\exp \left(\frac{\delta \pi}{\sqrt{1-\delta^{2}}}\right)\right)=\exp \left(\frac{\delta \pi}{\sqrt{1-\delta^{2}}}\right)
$$

y por lo tanto:

$$
A=\frac{1}{1+M_{p}}
$$

donde $M_{p}=\exp \left(\frac{-\delta \pi}{\sqrt{1-\delta^{2}}}\right)$ es el sobrepaso del sistema de segundo orden.

\begin{tabular}{|l|l|l|l|}
\hline$T$ & $A$ & $\delta_{i}$ & $w_{i}$ \\
\hline $0.069968 \mathrm{seg}$ & 0.500621 & 0.0355 & 44.9450 \\
\hline $0.991104 \mathrm{seg}$ & 0.509031 & 0.0364 & 3.1699 \\
\hline $63.83266 \mathrm{seg}$ & 0.961659 & 0.0505 & 0.0493 \\
\hline
\end{tabular}

Tabla 1: Valores de los parámetros del prefiltro.

Aplicando el prefiltro en el sistema mecánico de la grúa viajera, considerando la ubicación de los polos en lazo cerrado encontrados (21), se calcula el tiempo $T$ de la señal de entrada aplicada. 
G. Bonilla Mota, et al. $\quad$ Modelado y control de una grúa móvil con tres grados de libertad...

De igual manera se obtienen los valores de la amplitud $A(27)$, teniendo en consideración las $\delta_{i}, w_{i}=1,2, \ldots, 6$, de los polos en lazo cerrado, la tabla 1 muestra estos valores.

\section{Resultados}

Las simulaciones de la estrategia de control propuesta y del prefiltro se realizaron en MatlabSimulink, con los siguientes parámetros: $M=100, m_{1}=1, m_{2}=100, l_{1}=1, l_{2}=1$, $g=10, f=1000$ y con las siguientes condiciones iniciales $\left(x(0), \dot{x}(0), \theta_{1}(0), \dot{\theta}_{1}(0), \theta_{2}(0), \dot{\theta}_{2}(0)\right)=$ $(0,0,0,0,0,0)$. Hay que resaltar que no obstante que la ley de control se obtuvo a partir del modelo linealizado, las simulaciones se efectuaron sobre el modelo no-lineal original.

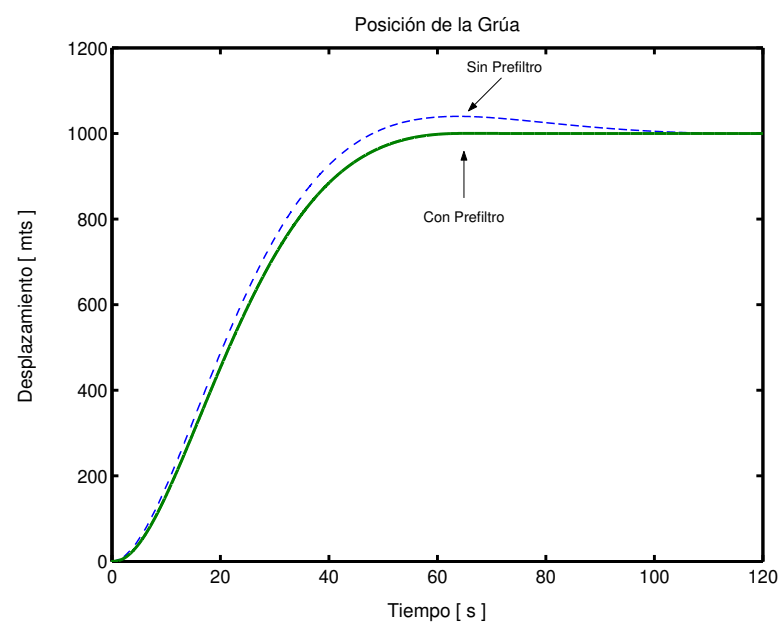

Figura 2: Comparación del comportamiento del desplazamiento usando LQR con y sin prefiltro.

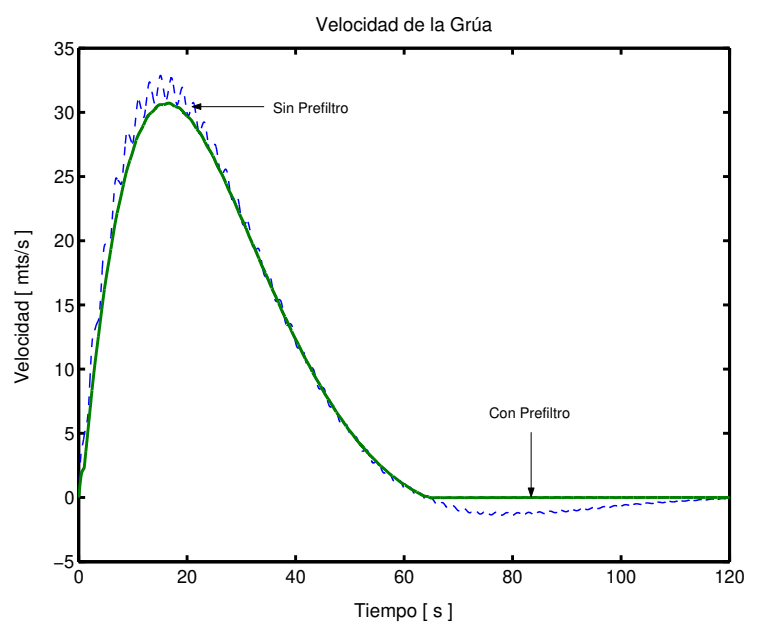

Figura 3: Comparación del comportamiento la velocidad usando LQR con y sin prefiltro.

A continuación se presenta el comportamiento del sistema con la ley de control LQR propuesta y se compara con el sistema al aplicar el prefiltro para apreciar el desempeño en ambos casos. La figura 2 compara el desplazamiento de la grúa con el control LQR propuesto con y sin el prefiltro. Se puede apreciar que el sistema converge al valor establecido y que el prefiltro reduce los transitorios que se observan con el control LQR. 
G. Bonilla Mota, et al. $\quad$ Modelado y control de una grúa móvil con tres grados de libertad...

En la figura 3 se presenta la comparación del comportamiento de la velocidad del sistema, de manera similar se reducen los transitorios. En las figuras 4 y 5 se presentan respectivamente, el comportamiento de $\theta_{1}$ y el de su velocidad.

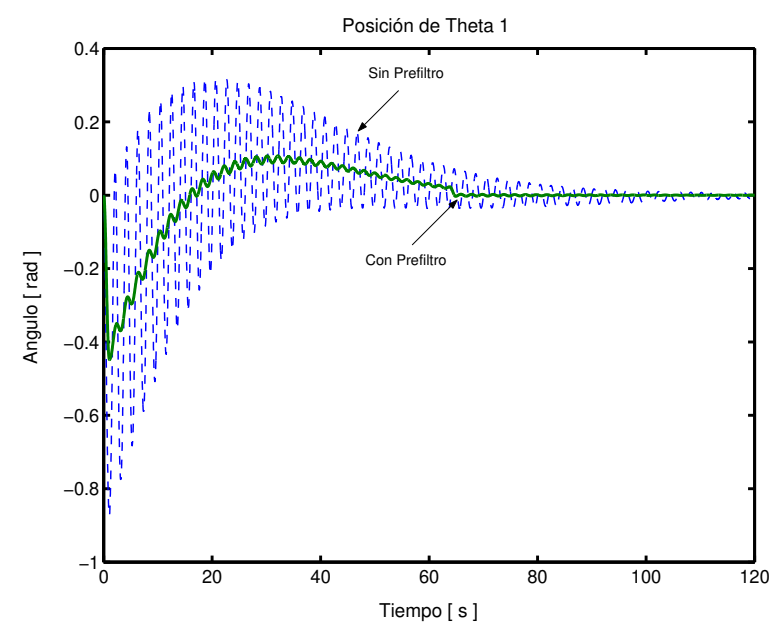

Figura 4: Comparación del comportamiento de $\theta_{1}$ usando LQR con y sin prefiltro.

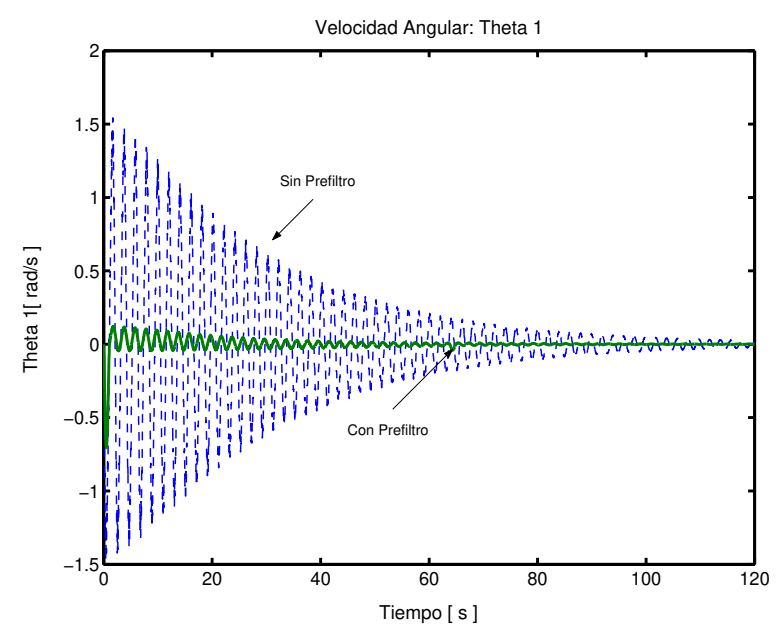

Figura 5: Comparación del comportamiento de la velocidad de $\theta_{1}$ usando LQR con y sin prefiltro.

El comportamiento de $\theta_{2}$ y de su velocidad se muestran en las figuras 6 y 7 , respectivamente.

De los resultados se concluye que el control LQR propuesto permite controlar el sistema mecánico de la grúa viajera y que la técnica del empleo de prefiltros redujo las oscilaciones de la respuesta transitoria del sistema. Es importante mencionar que el prefiltro requiere que el sistema se haya estabilizado previamente mediante algún controlador en malla cerrada.

\section{Conclusiones}

En este trabajo se realizó el estudio del sistema mecánico nonebbl@id@@englishid@@english subactuado de la grúa viajera constituida por un carro sobre una plataforma horizontal y un péndulo doble que se traslada de un punto inicial a otro final. Se obtuvo el modelo dinámico del sistema mediante ecuaciones diferenciales ordinarias no lineales y se diseñó una ley de control 
G. Bonilla Mota, et al. $\quad$ Modelado y control de una grúa móvil con tres grados de libertad...

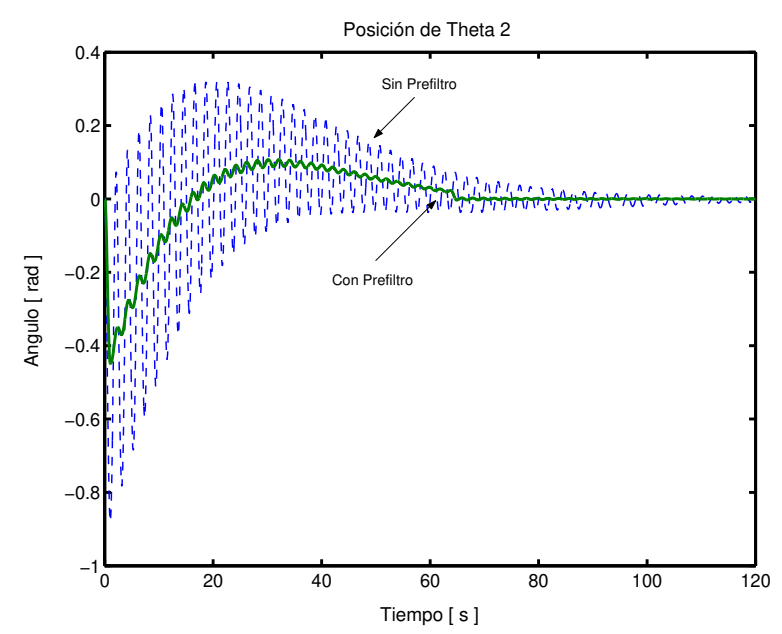

Figura 6: Comparación del comportamiento de $\theta_{2}$ usando LQR con y sin prefiltro.

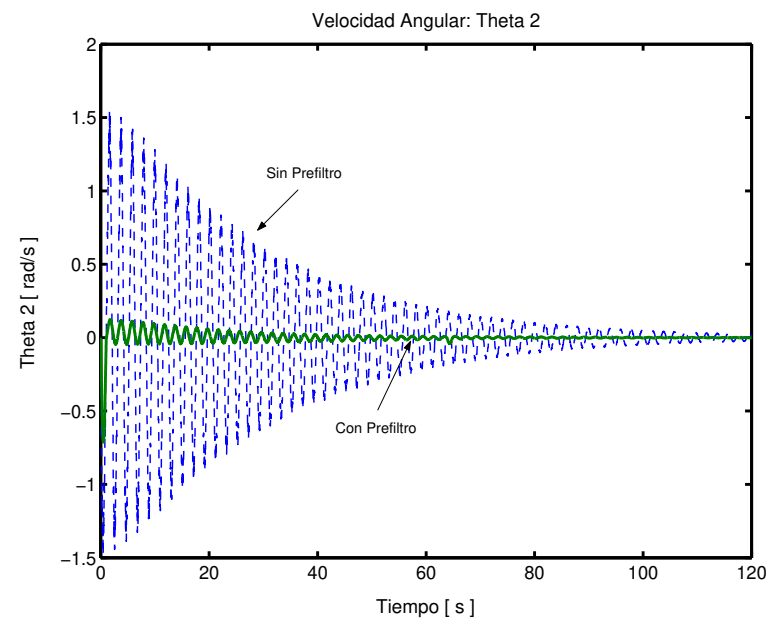

Figura 7: Comparación del comportamiento de la velocidad de $\theta_{2}$ usando LQR con y sin prefiltro.

LQR a partir del sistema linealizado. Además, se introdujo un prefiltro o precompensador en el sistema no lineal y se realizó una comparación de la estrategia de control con y sin prefiltro para determinar el desempeño del sistema. Los resultados obtenidos demuestran que el movimiento del péndulo doble logró controlarse. Por otra parte, el comportamiento del sistema original mejoró con el prefiltro, ya que se redujeron las oscilaciones en la respuesta del sistema. Por tanto, los prefiltros son una alternativa que se puede agregar a una ley de control estabilizante para mejorar su respuesta transitoria.

\section{Referencias}

[1] K. Krastanov, "About the safety by using mobile cranes," in The Euroasia Proceedings of Science, Technology, Engineering \& Mathematics (EPSTEM), vol. 1, pp. 213-217, 2017.

[2] R. L. Neitzel, N. S. Seixas, and K. K. Ren, "A review of crane safety in the construction industry," Applied Occupational and Environmental Hygiene, vol. 16, no. 12, pp. 1106-1117, 2001.

[3] M. W. Spong, "Underactuated mechanical systems," in Control Problems in Robotics and Automation. Lecture Notes in Control and Information Sciences (B. Siciliano and K. P. Valavanis, eds.), vol. 230, pp. 135-159, Springer, Berlin, Heidelberg, 1998. 
G. Bonilla Mota, et al. $\quad$ Modelado y control de una grúa móvil con tres grados de libertad...

[4] E. M. Abdel-Rahman, A. H. Nayfeh, and Z. N. Masoud, "Dynamics and control of cranes: A review," Journal of Vibration and Control, vol. 9, no. 10, pp. 487-495, 2016.

[5] J. Stergiopoulos and A. Tzes, "An adaptive input shaping technique for the suppression of payload swing in three-dimensional overhead cranes with hoisting mechanism," in IEEE Conference on Emerging Technologies and Factory Automation ETFA, Patras, Greece, pp. 565-568, 2007.

[6] K. A. Alghanim, K. A. Alhazza, and Z. N. Masoud, "Discrete-time command profile for simultaneous travel and hoist maneuvers of overhead cranes," Journal of Sound and Vibration, vol. 345, no. 9, pp. 47-57, 2015.

[7] K. Kawada, T. Yamamoto, and Y. Mada, "Gain scheduled PD sway control of a lifted load for a mobile crane," Control and Intelligent Systems, vol. 33, no. 1, pp. 48-54, 2005.

[8] B. Yang and B. Xiong, "Application of LQR techniques to the anti-sway controller of overhead crane," Advanced Materials Research, vol. 139-141, pp. 1933-1936, 2010.

[9] Z. Wu, X. Xia, and B. Zhu, "Model predictive control for improving operational efficiency of overhead cranes," Nonlinear Dynamics, vol. 79, no. 4, pp. 2639-2657, 2015.

[10] H. Chen, Y. Fang, S. Member, and N. S. Sun, "A swing constraint guaranteed MPC algorithm for underactuated overhead cranes," IEEE/ASME Transactions on Mechatronics, vol. 21, no. 5, pp. 25432555,2016 .

[11] J. Smoczek and J. Szpytko, "Particle swarm optimization-based multivariable generalized predictive control for an overhead crane," IEEE/ASME Transactions on Mechatronics, vol. 22, no. 1, pp. 258-268, 2017.

[12] J. H. Muhammad, J. Mohsin, O. G. Syed, I. Shadid, N. K. Muhammad, H. M. Mazhar, and A. Ishtiaq, "Jib system control of industrial robotic three degree of freedom crane using hybrid controller," Indian Journals of Science and Technology, vol. 9, no. 21, pp. 1-9, 2016.

[13] L. Lee, P. Huang, Y. Shih, T. Chiang, and C. Chang, "Parallel neural network combined with sliding mode control in overhead crane control system," Journal of Vibration and Control, vol. 20, pp. 749-760, 2014.

[14] L. Ranjbari, A. H. Shirdel, M. Aslahi-Shahri, S. Anbari, A. Ebrahimi, M. Darvishi, M. Alizadeh, R. Rahmani, and M. Seyedmahmoudian, "Designing precision fuzzy controller for load swing of an overhead crane," Neural Computing and Applications, vol. 26, no. 7, pp. 1555-1560, 2015.

[15] L. Ramlia, Z. Mohameda, A. M. Abdullahia, H. I. Jaafara, and I. M. Lazim, "Control strategies for crane systems: A comprehensive review," Mechanical Systems and Signal Processing, vol. 95, pp. 1-23, 2017.

[16] F. E. Cellier, Continuous system modeling. Spring-Verlag, New York, 1991.

[17] R. Sepulchre, M. Janković, and P. V. Kokotović, Constructive Nonlinear Control. Springer-Verlag, Great Britain, 1997.

[18] H. K. Khalil, Nonlinear systems. Prentice-Hall, Michigan State University, 1996.

[19] J. Collado, "Prefiltros y comportamiento transitorio," tech. rep., UANL, 2001.
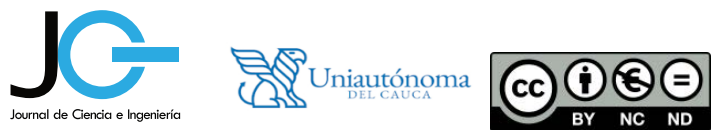